\title{
Preliminary Jiram Results from Juno Polar Observations: 1 - Methodology and Analysis Applied to the Jovian Northern Polar Region
}

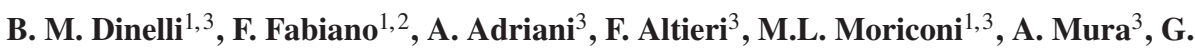

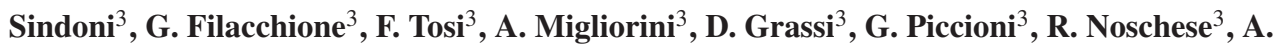 \\ Cicchetti $^{3}$, S.J. Bolton ${ }^{4}$, J.E.P. Connerney ${ }^{5}$, S.K. Atreya ${ }^{6}$, F. Bagenal ${ }^{7}$, G.R. Gladstone ${ }^{4}$, C. \\ Hansen $^{8}$, W.S. Kurth ${ }^{9}$, S.M. Levin ${ }^{10}$, B.H. Mauk ${ }^{11}$, D.J. McComas ${ }^{12}$, J.-C. Gèrard ${ }^{13}$, D.

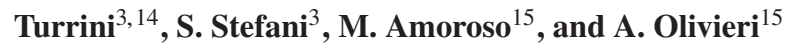 \\ ${ }^{1}$ ISAC-CNR - Via Gobetti,101 - Bologna - Italy \\ ${ }^{2}$ Dipartimento di Fisica e Astronomia, Università di Bologna - Bologna, Italy \\ ${ }^{3}$ IAPS-INAF, Via Fosso del Cavaliere, 100 - Roma - Italy \\ ${ }^{4}$ Southwest Research Institute, San Antonio, Texas, USA \\ ${ }^{5}$ NASA Goddard Space Flight Center, Greenbelt, Maryland, USA \\ ${ }^{6}$ University of Michigan, Ann Arbor, Michigan, USA \\ ${ }^{7}$ University of Colorado, Boulder, Colorado, USA \\ ${ }^{8}$ Planetary Science Institute, Tucson, Arizona, USA \\ ${ }^{9}$ Jet Propulsion Laboratory, California Institute of Technology, Pasadena, California, USA \\ ${ }^{10}$ University of Iowa, Iowa City, Iowa, USA \\ ${ }^{11}$ The Johns Hopkins University Applied Physics Laboratory, Laurel, Maryland, USA \\ ${ }^{12}$ Princeton University, Princeton, NJ 08544, USA \\ ${ }^{13}$ University of Liège, Liège, Belgium \\ ${ }^{14}$ Departamento de Fisica, Universidad de Atacama, Copayapu 485, Copiapò, Chile \\ ${ }^{15}$ Agenzia Spaziale Italiana, Roma, Italy
}

Key Points:

- First global maps of $H_{3}^{+}$intensity, column density and temperature for the Jupiter Northern aurora with high spatial resolution

- One side of the auroral oval shows higher $H_{3}^{+}$column density and lower temperatures in comparison with the other side

- Column densities main oval and temperature main oval do not superimpose

Corresponding author: Bianca Maria Dinelli, bm.dinelli@isac.cnr.it 


\section{Abstract}

During the first orbit around Jupiter of the NASA/JUNO mission, the Jovian Auroral InfraRed Mapper (JIRAM) instrument observed the auroral regions with a large number of measurements. The measured spectra show both the emission of the $\mathrm{H}_{3}^{+}$ion and of methane in the 3-4 $\mu \mathrm{m}$ spectral region. In this paper we describe the analysis method developed to retrieve temperature and Column Density (CD) of the $\mathrm{H}_{3}^{+}$ion from JIRAM spectra in the Northern auroral region. The high spatial resolution of JIRAM shows an asymmetric aurora, with CD and temperature ovals not superimposed and not exactly located where models and previous observations suggested. On the main oval averaged $\mathrm{H}_{3}^{+}$ CDs span between $1.8 \times 10^{12} \mathrm{~cm}^{-2}$ and $2.8 \times 10^{12} \mathrm{~cm}^{-2}$, while the retrieved temperatures show values between 800 and $950 \mathrm{~K}$. JIRAM indicates a complex relationship among $H_{3}^{+}$ CDs and temperatures on the Jupiter Northern aurora.

\section{Introduction}

In the Infra-Red (IR) spectral range Jupiter's aurora can be mapped thanks to the thermal emissions of the $\mathrm{H}_{3}^{+}$molecular ion, first detected by Drossart et al. [1989]. $\mathrm{H}_{3}^{+}$ forms at altitudes mainly above the Jovian homopause. At polar latitudes, accelerated energetic electrons that flow downward along magnetic field lines from the magnetosphere drive ionization of both atomic and molecular hydrogen [Atreya, 1986]. The ionised molecular hydrogen almost instantaneously react with $\mathrm{H}_{2}$ itself and create $\mathrm{H}_{3}^{+}\left(\mathrm{H}_{2}^{+}+\mathrm{H}_{2} \rightarrow \mathrm{H}_{3}^{+}\right.$ $+\mathrm{H})$. This is by far the major creation pathway for $\mathrm{H}_{3}^{+}$in planetary atmospheres; other minor creation routes involving $\mathrm{H}^{+}$and $\mathrm{H}_{2}$ are present but their effect is negligible with respect to the main process [Grodent et al., 2001]. In the upper atmosphere, where the density of free electrons is large, the $\mathrm{H}_{3}^{+}$ion is converted back to neutral hydrogen mainly by dissociative recombination $\left(\mathrm{H}_{3}^{+}+\mathrm{e}^{-} \rightarrow \mathrm{H}_{2}+\mathrm{HA} \hat{\mathrm{A}}\right.$ or $\left.\mathrm{H}_{3}^{+}+\mathrm{e}^{-} \rightarrow 3 \mathrm{H}\right)$. At lower altitudes, the main destruction pathway for $\mathrm{H}_{3}^{+}$is the ion-neutral charge exchange reactions with hydrocarbons (mainly $\mathrm{CH}_{4}$ and $\mathrm{C}_{2} \mathrm{H}_{2}$ ) producing molecular hydrogen and hydrocarbon ions.

The IR spectral range around $3.5 \mu \mathrm{m}$ is particularly suitable to study the $\mathrm{H}_{3}^{+}$emission as, in this spectral region, methane absorbs most of the light from the lower atmosphere of Jupiter and $\mathrm{H}_{3}^{+}$lines can be detected with a high contrast with respect to the dark planetary disk below. Auroral morphologies mapped through $\mathrm{H}_{2}$ UltraViolet (UV) 
and $\mathrm{H}_{3}^{+}$IR emissions are very similar on a global scale [e.g. Radioti et al., 2013] and three main components are usually identified [e.g. Clarke et al., 2004; Grodent, 2015]: the main oval, the polar emissions (poleward of the main emission), and the satellites footprints (equatorward of the main emission). However, unlike the auroral UV emissions that are a tracer of instantaneous energy inputs of the impacting electrons, the equatorward IR aurora also provides the information on the atmospheric response to the inputs. The surrounding neutral atmosphere quickly thermalises the $\mathrm{H}_{3}^{+}$ions after their formation. For this reason, $\mathrm{H}_{3}^{+} \mathrm{IR}$ emission lines can be used to derive the atmospheric temperature [e.g. Lam et al., 1997; Stallard et al., 2002], while integrated column densities retrieved using the intensities of the emission lines allow mapping the ion distribution.

On the NASA mission Juno, orbiting around Jupiter starting in early July 2016, the IR spectral range from 3 to $4 \mu \mathrm{m}$ was covered by the Jovian Infrared Auroral Mapper, JIRAM [Adriani et al., 2014]. This paper is the first of three papers where we report the observations of the Jupiter auroras made with the spectrometer of JIRAM: here we describe the methodology used to analyse JIRAM spectra of the auroral regions, and we report the results obtained for the Jupiter Northern polar region. Further results obtained for both the auroral regions are reported in Adriani et al. [this issue] and Moriconi et al. [this issue].

\section{JIRAM Instrument and Observations}

JIRAM [Adriani et al., 2014] is an imager/spectrometer designed to study the Jovian aurorae, as well as the planet's atmospheric structure, dynamics and composition. It is composed of two IR imager channels (M centered at $4.78 \mu \mathrm{m}$ and $\mathrm{L}$ centered at 3.45 $\mu \mathrm{m})$ and by a spectrometer. On the rows of the bi-dimensional spectrometer sensor, the entrance slit (with a Field Of View, FOV, of $3.5^{\circ}$ ) is covered by 256 pixels. The Instantaneous Field Of View (IFOV) of each pixel is about $250 \times 250 \mu \mathrm{rad}$. On the sensor column, the spectrum is sampled in 336 spectral channels in the $2-5 \mu \mathrm{m}$ range (mean spectral resolution about $9 \mathrm{~nm}$ ) with integration time of 30s. Thanks to the motion of the spacecraft and the JIRAM de-spinning mirror, each slit of the spectrometer is combined to provide a hyperspectral image (so called "image cube"), being the X-dimension of the cube provided by the slit (corresponding to the cross-track direction), the Y-dimension collected along-track, and the $\lambda$-dimension provided by the spectrometer spectral range. Data acquired during the Moon flyby of October 2013 and during the first perijove demon- 
strated the JIRAM radiometric performances [Adriani et al., 2016]. As already mentioned, in this work we focus on the spectrometer data in the $3-4 \mu \mathrm{m}$ sub-range.

Figure 1 shows a collage of all the analyzed measurements of the northern auroral region. As described in section 3.2 only measurements with emission angle lower than $75^{\circ}$ have been included in the figure. Panels $\mathbf{a}, \mathbf{b}$ and $\mathbf{c}$ of Figure 1 show Jupiter orthographic map in planetocentric coordinates focussed on the North auroral region with superimposed spots at the geo-location of the intercept of the Line Of Sight (LOS) of each spectrum acquired by JIRAM with the surface located $500 \mathrm{~km}$ above Jupiter 1 bar surface. The colour of each dot indicates the value of the represented quantity, while the size of the spot represents its spatial resolution. Panel a shows the emission angle of the measurements, that is the angle made by the LOS with the vertical to the $500 \mathrm{~km}$ altitude surface. It indicates that the measurements have been made observing the same part of Jupiter from different directions, and the measurements with higher spatial resolution are also the ones with lower emission angle. Panel $\mathbf{b}$ shows the solar incidence angle, that is the Solar Zenith Angle (SZA) at the intercept of the LOS with the $500 \mathrm{~km}$ surface. We see that measurements of both the dark and illuminated regions are available. Panel $\mathbf{c}$ shows the integrated intensities of the analysed measurements, obtained integrating the recorded signal over the 3.35-3.75 $\mu \mathrm{m}$ spectral region (where most of $\mathrm{H}_{3}^{+}$emission is located and no interferences with other molecules are present) and multiplying it by the cosine of the emission angle, to correct for the slant optical path. The orthographic surface shown in the panels has been divided in squared bins, obtained dividing each axis in regular intervals. The single intensities have been averaged over each bin, and bins containing less than 3 measurements have not been included in the final dataset. Panel d of Figure 1 represents the contour plot of the binned distribution. In all panels, the dashed line represents the geolocation of the auroral oval from existing models [Connerney et al., 1998] and the solid line is the statistical geolocation of the aurora [Bagenal et al., 2014]. In panel $\mathbf{d}$ we identify a region where the signal is maximum (that indicates a strong $\mathrm{H}_{3}^{+}$emission) and that falls close to the two auroral ovals. 


\section{$3 \mathrm{H}_{3}^{+}$temperature and column density estimation}

\subsection{Retrieval Code}

Temperature and column density of $\mathrm{H}_{3}^{+}$in the auroral regions have been determined by analysing JIRAM data in the 3.5 micron region. For this purpose, we have used an update of the code previously developed for the analysis of Galileo/NIMS spectra [Altieri et al., 2016]. Here we recall the basics of this system. The code is divided into two modules: the Forward Model (FM) and the Retrieval Module (RM). The FM is used to simulate the spectra measured by JIRAM. The spectra are simulated by assuming that the emission of the auroral region is optically thin, which is supported by the fact that the $\mathrm{H}_{3}^{+}$ layer is located in the highest part of Jupiter's atmosphere. We also assume that the pressure broadening of the spectral lines is negligible and that we can use the same temperature for all the lines of each gas. The latter hypothesis is justified by the fact that most of the auroral emission originates at altitudes where vibrational local thermal equilibrium can be safely assumed [Melin et al., 2005], and may produce a maximum error of 5\%. The spectrum is simulated by first computing the intensity of each transition of the gases that we want to include into the simulation. The intensity of the transition $k$ of the molecule $m$ can be computed using the expression reported by Altieri et al. [2016] taken from Stallard et al. [2002, and references therein].

The intensities are computed for all the spectral lines of the considered spectral region. To reproduce the measurements, the computed intensities are then convolved with the instrumental spectral response. Since part of the JIRAM data were acquired on the dayside, to take into account a variable background emission introduced by the scattered sunlight, we have introduced in the FM the possibility to add a radiometric offset, constant over the whole analysed spectral region, to the simulated spectra. The FM also includes the possibility to evaluate analytically the derivatives of the spectra with respect to temperature and column density of each gas.

The RM is the part that takes care of the determination of the required parameters. We invert the measured spectra using an iterative Bayesian approach. The parameters that can be retrieved with the RM are the column density along the instrument LOS and the effective temperature of each considered gas. Moreover, to account for spectral calibration problems, we can also retrieve a wavelength shift, the width of the instrumental response function, and the radiometric offset value. At each iteration, we evaluate the weighted $\chi^{2}$ 
$\left(\chi\right.$-test $\left.=n^{T} S^{-1} n\right)$ and the loop is stopped when two consecutive iterations do not yield values that differ for more than $1 \%$ percent.

\subsection{Analysis}

The targets of our analysis are the effective temperature of $\mathrm{H}_{3}^{+}$and its column density along the Line Of Sight (LOS) of each observation. Since in recent studies [see $\mathrm{Al}$ tieri et al., 2016, and reference therein] methane emissions have been detected in the auroral region, along with the $\mathrm{H}_{3}^{+}$data we may simultaneously retrieve temperature and column density of methane along the LOS.

$\mathrm{H}_{3}^{+}$transitions and spectral properties have been downloaded from the web site http://www.tampa.phys.ucl.ac.uk/ftp/astrodata/H3+/ [Neale et al., 1996] and the partition function has been computed using the expression of Miller et al. [2013]. $\mathrm{CH}_{4}$ spectroscopic data have been taken from the HITRAN 2012 database [Rothman et al., 2013] and its partition function is evaluated using the routine of Gamache described in Laraia et al. [2011]. In this work, JIRAM instrumental response function is assumed to be a Gaussian function whose width has been evaluated during the on-ground calibration campaign [Adriani et al., 2014], therefore we did not include in the retrieval the corresponding parameter.

Figure 2 shows two JIRAM spectra in the $\mathrm{H}_{3}^{+}$emission spectral region, one acquired in the sunlit part and one in the dark part of the northern aurora. As can be seen in the Figure, the spectral region below $3.2 \mu \mathrm{m}$ is affected by a continuum due to the scattering of sunlight by the lower atmospheric layers, that cannot be corrected using a simple expression. The spectral region above $3.8 \mu \mathrm{m}$ is contaminated by both instrumental effects and scattered radiation. Therefore, to avoid systematic errors due to the poor representation of the measured spectrum, we have restricted our analysis to the 3.2-3.8 $\mu \mathrm{m}$ region.

During the first Juno orbit, on Aug. $27^{\text {th }} 2016$ in total JIRAM acquired about 75000 spectra when observing the North Polar region of Jupiter. More than 16000 were recorded over the North auroral region from 08:24 to 11:51 UTC. Those spectra were acquired at different times, and both the emission angle and the pixel size at Jupiter span a wide variety of values. Therefore, in order to properly map the emission on Jupiter disk, only spectra with an emission angle smaller than $75^{\circ}$ have been retained in the analysis. However some of the selected spectra were affected by strong intensity spikes or showed an $\mathrm{H}_{3}^{+}$sig- 
nal too weak to produce a reliable retrieval. Given the number of measured spectra, an automatic procedure has been designed to perform a pre-filtering of the measurements. The first step of this procedure was the identification of the spikes produced by energetic particles on the detector. For each spectrum, we have evaluated the maximum intensity recorded at the wavelengths of the $\mathrm{H}_{3}^{+}$lines. All the spectral points outside the $\mathrm{H}_{3}^{+}$lines whose intensity was larger than 1.5 times the maximum intensity were flagged as spikes, and masked out from the retrieval. Spectra with 3 or more spikes were completely discarded. The second step, applied after the spike removal, was the removal of the spectra where the $\mathrm{H}_{3}^{+}$signal was below the detection limit, set to $0.0001 \mathrm{~W} / \mathrm{m}^{2} / \mathrm{sr}$. The final set of measurements included a total of 14131 spectra.

Each spectrum in the final set of measurements has been analysed with the retrieval code described in section 3.1. In the first run we considered $\mathrm{H}_{3}^{+}$only in the simulated spectra and the target parameters were: $\mathrm{H}_{3}^{+}$effective temperature (T) and column density (CD), a wavelength shift and an offset value for each spectrum. The uncorrelated a-priori errors (diagonal $S_{a}$ ) used in the retrieval were chosen to ensure a very small constraint on the retrieval results. For all the spectra we have assumed the same Noise Equivalent Spectral Radiance (NESR) of $1.5 \times 10^{-7} \mathrm{~W} /\left(\mathrm{m}^{2} \mathrm{~nm} \mathrm{sr}\right)$, evaluated from deep space spectra.

A first inspection of the retrieval results highlighted that the spectra acquired over the region inside the auroral oval showed higher $\chi$-test values than the other spectra and anomalous $\mathrm{H}_{3}^{+}$temperatures. A visual inspection of some of these spectra showed that the intensity of the $\mathrm{H}_{3}^{+}$line at $3.32 \mu \mathrm{m}$ was always too high in comparison to other $\mathrm{H}_{3}^{+}$diagnostic lines. Considering that methane has already been observed in Jupiter auroral region [Altieri et al., 2016] and its $v_{3}$ Q-branch lies in the same spectral region, we simulated the analysed spectral region adding the methane emission around $3.3 \mu \mathrm{m}$. A quick comparison of the simulated spectra with our measurements showed that the recorded signal was compatible with the $\mathrm{CH}_{4}$ emission at $500 \mathrm{~K}$ superimposed to the $\mathrm{H}_{3}^{+}$spectrum [see Moriconi et al., this issue]. We therefore repeated the analysis of all the spectra including the $\mathrm{CH}_{4}$ column density among the target parameters, keeping its effective temperature fixed at $500 \mathrm{~K}$. Indeed this inclusion reduced the $\chi$-test value of the retrieval. In the regions where $\mathrm{CH}_{4}$ emission was not a dominant feature, the inclusion of methane did not change significantly the results of the fit for the $\mathrm{H}_{3}^{+}$parameters. Where the methane emission was significant, the new $\mathrm{H}_{3}^{+}$temperatures assumed values in the expected range (700-1100 K). Finally, since the wavelength calibration of the measured spectra is expected to be depen- 
dent only from the position of the pixel on the spectrometer slit, we used the results of the first run to fit a second order polynomial function to the set of retrieved wavelength shift versus position of the pixel. We then used that polynomial to compute the real wavelength scale and repeated the analysis (final analysis) using as free parameters just T, $\mathrm{CD}, \mathrm{CH}_{4}$ column density and the offset. This was done to prevent the retrieval code to use the frequency shift to partially correct for other instrumental problems and therefore producing a bias in the retrieved parameters.

The results of the final analysis were further filtered by retaining only the retrievals of the spectra for which the final $\chi$-test was smaller than 20 , and the obtained $\mathrm{T}$ had a retrieval error lower than $100 \mathrm{~K}$. No filter was applied to the size of the error on the $\mathrm{H}_{3}^{+}$ CDs. The final number of obtained results is 13198 .

\section{$4 \mathrm{H}_{3}^{+}$results}

As can be seen in panel a of Fig. 1, the emission angle of the analysed observations spans a wide range of values. The retrieved CD is proportional to the length of the optical path inside the $\mathrm{H}_{3}^{+}$layer (slant columns). These two facts make impossible a direct comparison of the retrieved CDs. Therefore we have transformed all the retrieved slant columns into vertical columns multiplying the retrieved values by the cosine of the emission angles of the corresponding observation. Then, assuming that the vertical extent of the $\mathrm{H}_{3}^{+}$layer at a certain geo-location is constant, similarly to what we have already done for the integrated intensities (see section 2), we have divided Jupiter surface into bins and we have averaged all the retrieval results and their errors inside the bins. The results are reported in Figure 3 for the column densities and in Figure 4 for the temperatures.

The right panel of Figure 3 shows that on average the retrieval error on the CD is below $30 \%$ and that the error is lower where the CDs assume the highest values. The left panel of Figure 3 shows that the peak of $\mathrm{H}_{3}^{+}$column densities lays in part above the model oval (dashed line) and in part closer to the statistical oval (solid line). The right panel of Figure 4 shows that the highest errors on $\mathrm{T}$ are located in the region inside the auroral oval and in general where the $\mathrm{H}_{3}^{+}$signal is lower (see panel $\mathbf{d}$ of Figure 1 for comparison). The left panel of Figure 4 shows that in general the highest temperatures are located on the left side of the auroral region. 
Comparing the left panels of Figure 3 and 4 we have identified 3 regions of interest, highlighted in Fig. 5:

A: Longitudes from $200^{\circ}$ to $240^{\circ}$ and latitudes from $90^{\circ}$ to $65^{\circ} \mathrm{N}$. This region of the main auroral oval is characterised by high CDs inside (poleward of) the statistical oval with a peak (CD larger than $3.0 \times 10^{12} \mathrm{~cm}^{-2}$ ) in the longitude range from $200^{\circ}$ to $210^{\circ}$ and $67^{\circ} \mathrm{N}$ in latitude. The corresponding temperature is about $850 \mathrm{~K}$ on average. Higher values for the temperatures are retrieved equatorward, in the region located between the model and the statistical oval. A high CD region (with values about 2 times lower) was observed at the same latitudes by Miller et al. [1997] with the United Kingdom Infrared Telescope. Similarly to what we find, the corresponding $\mathrm{H}_{3}^{+}$temperatures were of the order of $880-900 \mathrm{~K}$. The morphological analysis in Mura et al. [this issue] made using the imager L channel of JIRAM (3.3-3.6 $\mu \mathrm{m})$ shows that this is a region of broad emission with thin coherent features (arcs) that are visible from the main oval to 10 degrees inward. Such region may be still magnetically connected to the equatorial plane; this is in agreement with our finding of uniform temperature and $\mathrm{CD}$.

B: Longitudes from $60^{\circ}$ to $95^{\circ}$, latitudes from $90^{\circ}$ to $75^{\circ} \mathrm{N}$. On the oval arc crossing the pole, $H_{3}^{+}$CDs show variation between 2 and $2.6 \times 10^{12} \mathrm{~cm}^{-2}$, with a peak on the North Pole. Temperatures show values between 800 and $850 \mathrm{~K}$, with a peak eastward of the North Pole of about 900 K.

C: Longitudes from $90^{\circ}$ to $160^{\circ}$, latitudes from $80^{\circ}$ to $60^{\circ} \mathrm{N}$. In this region higher CD values (larger then $2.6 \times 10^{12} \mathrm{~cm}^{-2}$ ) are retrieved external to the statistical oval. Temperatures show high variability between 800 and $950 \mathrm{~K}$, with peaks on the statistical oval. UV emission increases have been also found in previous UV data [e.g. Clarke et al., 2004; Clarke, 2013], and have been associated to 'dawn storms'. Moreover this side of the oval appears narrower than the other side. The shape of the auroral oval in this region, as seen also in the images reported by [Mura et al., this issue], appears extremely sharp.

We also notice that there are spots where the temperatures are very high. Spotty features have been identified also in the L-band images reported by [Mura et al., this issue]. The first extensive mapping of $\mathrm{H}_{3}^{+}$temperatures and $\mathrm{CDs}$ in the Northern Aurora of Jupiter, reported in this paper, highlight many differences in their morfology. The 
$\mathrm{T}$ oval and the $\mathrm{CD}$ oval appear asymmetric and tilted one with respect to the other. In general regions with high $\mathrm{H}_{3}^{+}$CDs do not coincide with regions with high T. Following O'Donoghue et al. [2014], we can speculate that high energy electrons penetrate deep in the atmosphere producing large $\mathrm{H}_{3}^{+}$quantities at low altitudes, where temperatures are low, while less energetic electrons will stop at higher altitudes, generating larger $\mathrm{H}_{3}^{+}$quantities in the upper layers of the atmosphere, where the temperature is larger. Assuming the auroral temperature profile reported by Grodent et al. [2001], a $100 \mathrm{~K}$ difference (from $950 \mathrm{~K}$ to $850 \mathrm{~K}$ ) would mean a difference of about $150 \mathrm{~km}$ in the penetration depth of the electrons. If this is effectively the case, the temperature/CD maps would give some hint on the variability of precipitating electron energies, as suggested by Hiraki and Tao [2008]. Another possibility is that the lower temperatures observed in correspondence of large CDs are a signature of the " $\mathrm{H}_{3}^{+}$thermostat effect" [Miller et al., 2013]: larger $\mathrm{H}_{3}^{+}$densities lead to an increased infrared cooling of the atmosphere that lowers the local temperature. However, we do not observe a strict anti-correlation between temperature and $\mathrm{CD}$, suggesting that the thermostat mechanism is not enough to explain the observed differences.

\section{Conclusions}

We have developed an analysis tool to invert JIRAM spectra in the $3-4 \mu \mathrm{m}$ spectral region to retrieve informations on $\mathrm{H}_{3}^{+}$distribution and temperatures in Jupiter auroral regions. The tool has been applied to JIRAM measurements acquired over the North Polar region during the first Juno orbit around Jupiter. Given the number of measurements acquired by JIRAM, an automatic procedure to identify and discard problematic measurements has been developed. We have evaluated the distribution of the $\mathrm{H}_{3}^{+}$column densities and temperatures in the Northern aurora. The analysis shows that the location of the maximum $\mathrm{H}_{3}^{+}$concentration is close to what models and previous observations suggest. The high spatial resolution of the measurements suggest that the North aurora of Jupiter is not uniform, with different distributions of the $\mathrm{H}_{3}^{+}$abundance and temperature. On the main oval averaged $H_{3}^{+}$CDs span between $1.8 \times 10^{12} \mathrm{~cm}^{-2}$ and $2.8 \times 10^{12} \mathrm{~cm}^{-2}$, while the retrieved temperatures show variation between 800 and $950 \mathrm{~K}$. On the auroral region at longitudes from $90^{\circ}$ to $170^{\circ}$ higher $\mathrm{H}_{3}^{+}$column densities are observed equatorward of the main oval, whereas the temperature is higher inside the statistical oval region. On the contrary, in the auroral region from $200^{\circ}$ to $210^{\circ}$ in longitude higher temperatures are ob- 
served with increasing values equatorward. JIRAM first data confirm the complex relationship among $\mathrm{H}_{3}^{+}$emission rates, CDs and temperatures on the Jupiter Northern aurora.

\section{Acknowledgments}

The JIRAM project has been funded by the Italian Space Agency contract number 201623-H.0. The data reported in this paper are available upon request to the main author.

\section{References}

Adriani, A., et al. (2014). JIRAM, the Jovian Infrared Auroral Mapper Space Science Reviews 1-54. doi:10.1007/s11214-014-0094-y

Adriani, A., M.L. Moriconi, A. Mura, F. Tosi, G. Sindoni, R. Noschese, A.Cicchetti, G. Filacchione (2016). Juno's Earth flyby: The Jovian infrared Auroral Mapper preliminary results Astophys. Space Sci., 361-272, doi:101007/s10509-016-2842-9

Adriani, A., et al. (2017). Preliminary JIRAM Results from Juno Polar Observations: 2 Analysis of the Jupiter Southern $\mathrm{H}_{3}^{+}$emissions and Comparison with the North Aurora. , This issue

Altieri, F., B. M. Dinelli, A. Migliorini, M. L. Moriconi, G. Sindoni, A. Adriani, A. Mura, and F. Fabiano (2016), Mapping of hydrocarbons and $\mathrm{H}_{3}^{+}$emissions at Jupiter's north pole using Galileo/NIMS data Geophys. Res. Lett., 43, 11,558-11,566, doi:10.1002/2016GL070787.

Atreya, S. K. (1986). Atmospheres and Ionospheres of the Outer Planets and their Satellites, pp 106-144, Springer-Verlag, New York-Berlin-Heidelberg.

Connerney, J. E. P., M. H. Acuña, N. F. Ness, and T. Satoh (1998), New models of Jupiter's magnetic field constrained by the Io flux tube footprint, J. Geophys. Res., 103(A6), 11,929-11,939, doi:10.1029/97JA03726

Bagenal F.,A. Adriani, F. Allegrini, S.J. Bolton, B. Bonfond, E.J. Bunce, J.E.P. Connerney, S.W.H. Cowley, R.W. Ebert, G.R. Gladstone, C.J. Hansen, W.S. Kurth, S.M. Levin, B.H. Mauk, D.J. McComas, C.P. Paranicas, D. Santos-Costa, R.M. Thorne, P. Valek, J.H. Waite, P. Zarka, (2014) Magnetospheric Science Objectives of the Juno Mission, Space Sci Rev, doi:10.1007/s11214-014-0036-8.

Clarke, J. T., et al. (2004), Jupiter's Aurora, in Jupiter, the Planet, Satellites and Magnetosphere, pp. 639-670, Cambridge Univ. Press, Cambridge, U. K. 
Clarke, J.T., (2013) Auroral Processes on Jupiter and Saturn, in Auroral Phenomenology and Magnetospheric Processes: Earth And Other Planets, pp.113-122, American Geophysical Union, doi:10.1029/2011GM001199

Drossart, P. et al.Âă (1989). Detection of $\mathrm{H}_{3}^{+}$on Jupiter, Nature, 340, 539-541, doi:10.1038/340539a0

Grodent, D., Waite J.H. Jr., Gèrard J.-C., (2001) A self-consistent model of the Jovian auroral thermal structure, J. Geophys. Res.- Space Phys., 106,12933-12952, doi:10.1029/2000JA900129.

Grodent, D., (2015) A brief review of ultraviolet auroral emissions on giant planets, Space Sci. Rev., 187, 23-50, doi:10.1007/s11214-014-0052-8.

Y. Hiraki and C. Tao (2008), Parameterization of ionization rate by auroral electron precipitation in Jupiter, Ann. Geophys., 26, 77-86, doi:10.5194/angeo-26-77-2008

Lam, H. A., N. Achilleos, S. Miller, J. Tennyson, L. M. Trafton, T. R. Geballe, and G. E. Ballester (1997), A baseline spectroscopic study of the infrared auroras of Jupiter, Icarus, 127, 379-393, doi:10.1006/icar.1997.5698.

Laraia, A. L., R. R. Gamache, J. Lamouroux, I. E. Gordon, and L. S. Rothman (2011), Total internal partition sums to support planetary remote sensing, Icarus, 215, 391-400, doi:10.1016/j.icarus.2011.06.004.

Melin, H., S. Miller, T. Stallard, D. Grodent (2005), Non-LTE effects on $\mathrm{H}_{3}^{+}$emission in the jovian upper atmosphere, Icarus, 97-103, doi:10.1016/j.icarus.2005.04.016

Miller, S., Achilleos, N., Ballester, G. E., Lam, H. A., Tennyson, J., Geballe, T. R., Trafton, L. M. (1997) Mid-to-low latitude $\mathrm{H}_{3}^{+}$emission from Jupiter. Icarus, 130, 57 67, doi:10.1006/icar.1997.5813

Miller, S., T. Stallard, J. Tennyson, and H. Melin (2013), Cooling by $\mathrm{H}_{3}^{+}$Emission, J. Phys. Chem. A, 117, 9770-9777, doi:10.1021/jp312468b

Moriconi, M.L., et al. (2017) Preliminary JIRAM Results from Juno Polar Observations: 3 - Evidence of Diffuse Methane Presence in the Jupiter Auroral Regions, This issue

Mura., A. et al. (2017) Auroral emission detected by JIRAM L band inager channel during first Juno orbit ,This issue

Neale, L., S. Miller, and J. Tennyson (1996), Spectroscopic properties of the $\mathrm{H}_{3}^{+}$molecule: A new calculated line list, Astrophys. J., 464, 516-520, doi:10.1086/177341

O'Donoghue, J. et al. (2014) Conjugate observations of Saturn's northern and southern $\mathrm{H}_{3}^{+}$ aurorae, Icarus, 229, 214-220, doi:/10.1016/j.icarus.2013.11.009 
Radioti, A., M. Lystrup, B. Bonfond, D. Grodent, and J.-C. Gèrard (2013), Jupiter's aurora in ultraviolet and infrared: Simultaneous observations with the Hubble Space Telescope and the NASA Infrared Telescope Facility, J. Geophys. Res. Space Physics, 118, 22862295, doi:10.1002/jgra.50245.

Rothman, L. S., et al. (2013), The HITRAN2012 molecular spectroscopic database, J. Quant. Spectros. Radiat. Transfer, 130, 4-50, doi:10.1016/j.jqsrt.2013.07.002

Stallard, T., S. Miller, G. Millward, and R. D. Joseph (2002), On the dynamics of the Jovian ionosphere and thermosphere: II. The measurement of $\mathrm{H}_{3}^{+}$vibrational temperature, column density, and total emission, Icarus, 156, 498-514, doi:10.1006/icar.2001.6793. 


\section{$270^{\circ}$}

Figure 1. Orthographic map of Jupiter North Pole in planetocentric coordinates with superimposed spots at the geo-location of the intercept of JIRAM LOS with the surface located at $500 \mathrm{~km}$ above Jupiter 1-bar surface. The longitude scale is reported above the axis and dashed circles have been drawn every $10^{\circ}$ of latitude. The colour of each dot indicates the value of the represented quantity, while the size of the spot represents its spatial resolution. The black lines represent the position of the aurora from models (dashed line) and from statistics (solid line) Panel a shows the emission angle of the measurements, panel b shows the solar incidence angle, panels $\mathbf{c}$ and $\mathbf{d}$ show the integrated intensities of the measurements. 
Figure 2. JIRAM spectra acquired in the sunlit auroral region (red line Jupiter solar time 6:36) and in the 
Figure 3. Same region of Figure 1. Left panel: map of the retrieved $\mathrm{H}_{3}^{+}$column densities. Right panel: map of the average retrieval error on the retrieved CDs. 
Figure 4. Same region of Figure 1. Left panel: map of the retrieved $\mathrm{H}_{3}^{+}$effective temperatures. Right panel: map of the average retrieval error on the retrieved Ts. 
Figure 5. Comparison of the distribution of $\mathrm{H}_{3}^{+} \mathrm{CD}$ (left panel) and $\mathrm{T}$ (right panel).

$-18$ 
'Figure 1'. 

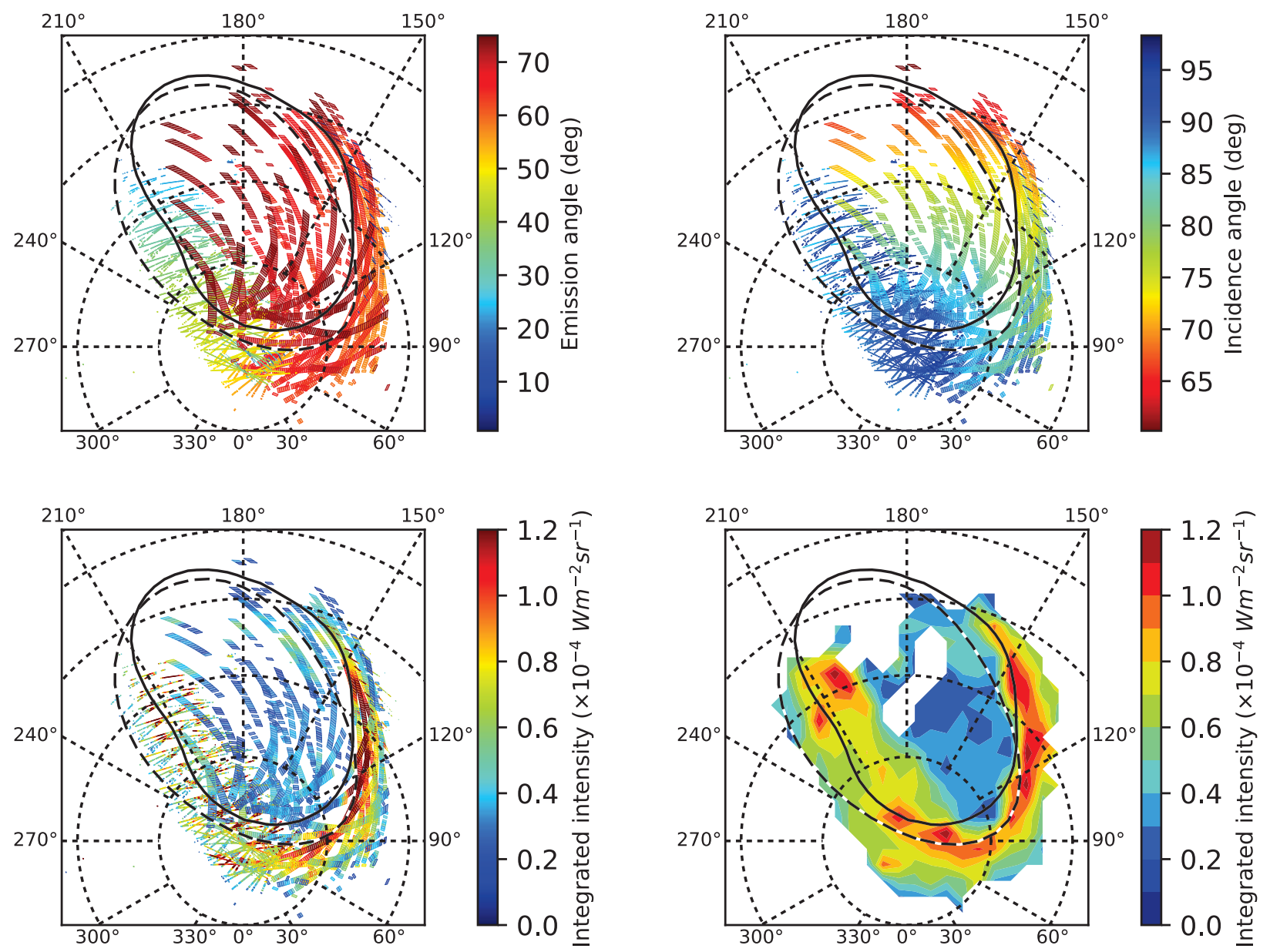
'Figure 2'. 


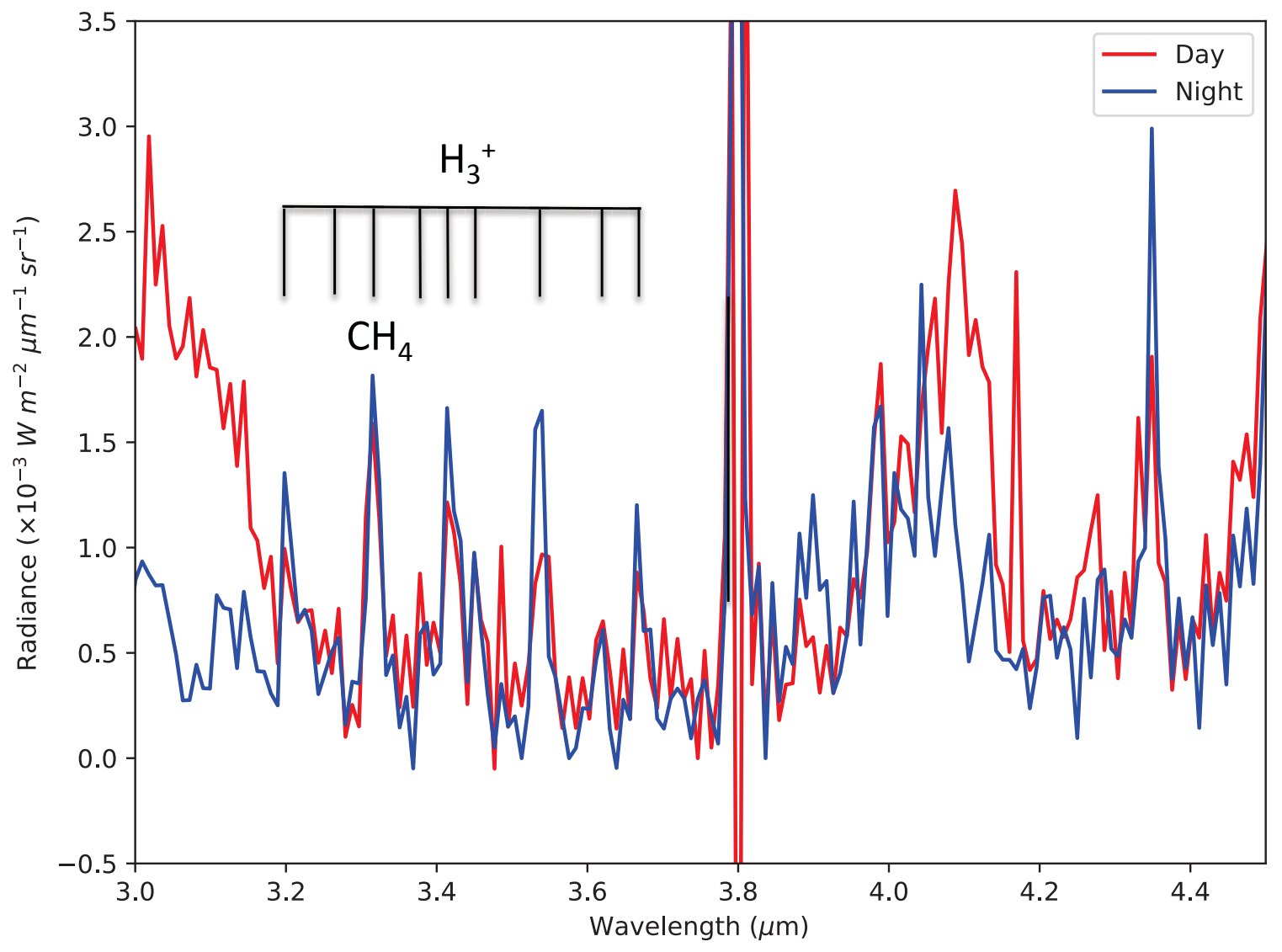


'Figure 3'. 

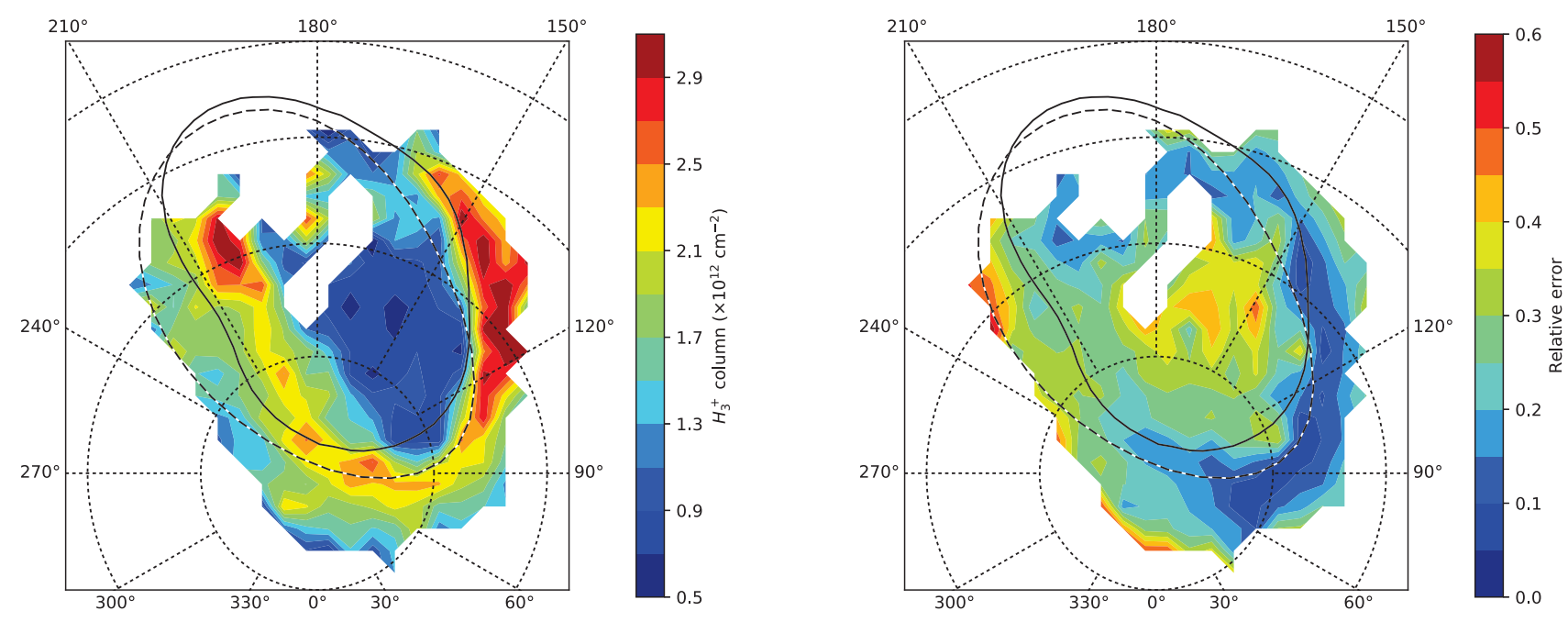
'Figure 4'. 

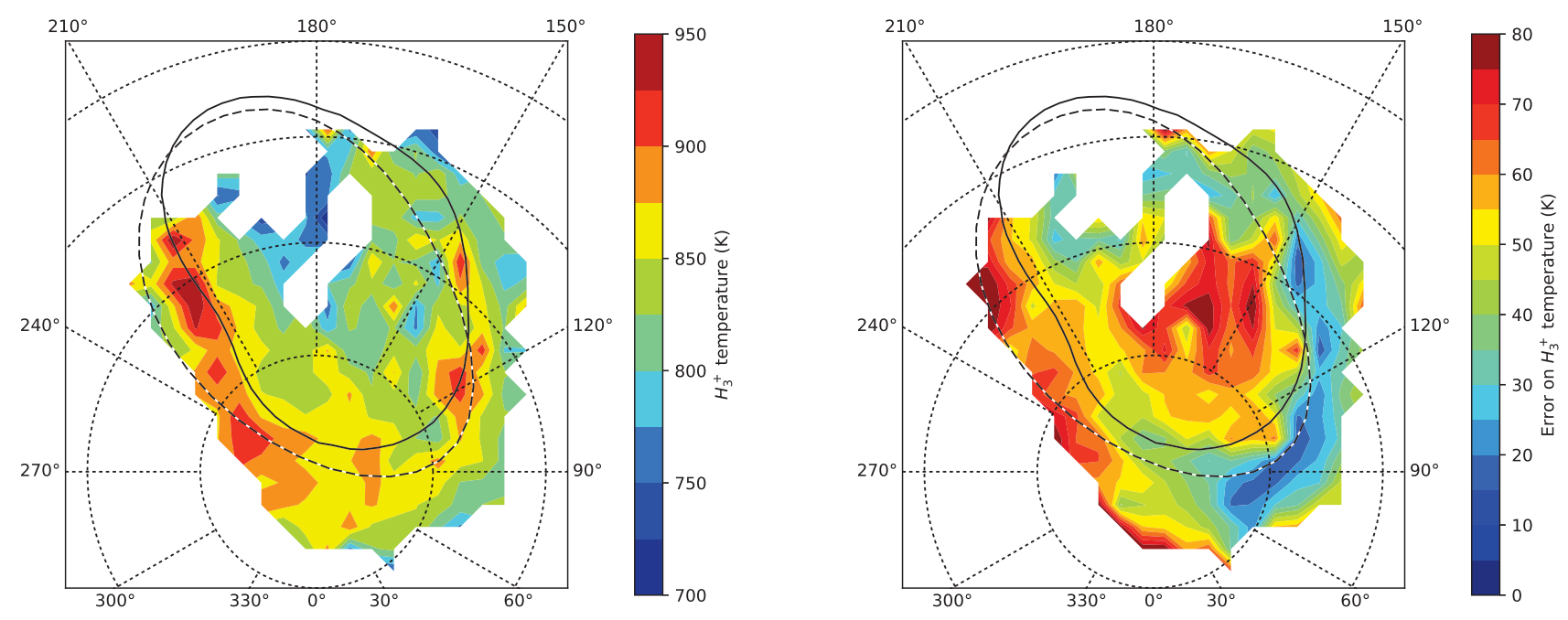
'Figure 5'. 

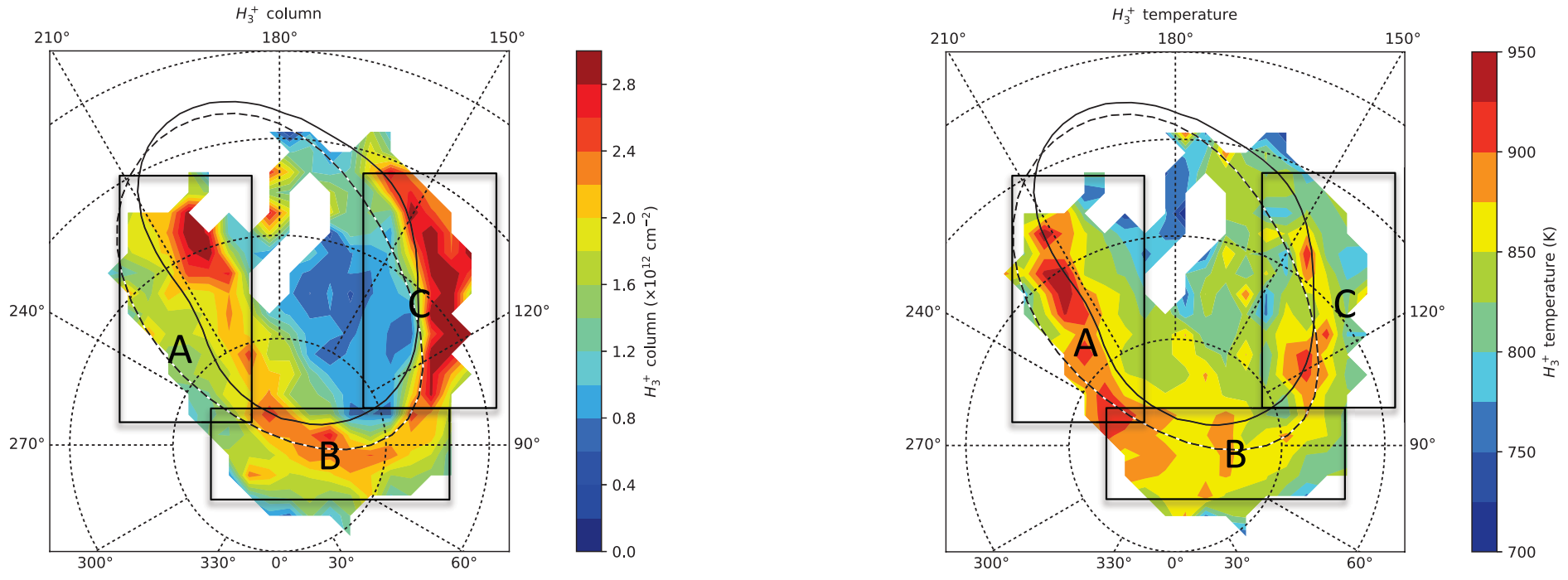